\title{
Application of Fourier algorithm to Near Field Optical Images: Local Resolution Estimation
}

\author{
Dominique Barchiesi \\ Laboratoire d'Optique P.M. Duffieux, URA CNRS 214, Université de Franche-Comté, \\ 13 route de Gray, 25030 Besançon Cedex, France
}

(Received October 4; accepted October 18, 1996)

PACS.42.30.Kq - Fourier optics

PACS.42.30.Va - Image forming and processing

\begin{abstract}
In Near Field Optics, the lateral resolution is better than the limit given by the Rayleigh criterion, but this fact was usually demonstrated by using test samples. In this study we apply local Fourier algorithms to near field optical experimental images, using a noise consideration to define resolution criterion. These algorithms are able to estimate the resolution automatically and thus can lead to simple image processing. We investigate the local resolution in scanning lines and we introduce the apparatus characterization. In the first part we discuss on the problem of resolution in near field optics.
\end{abstract}

Résumé. - En optique de champ proche, la résolution latérale dépasse la limite donnée par le critère de Rayleigh, mais ce fait est généralement démontré en utilisant des objets tests. Dans cette étude, nous appliquons localement des algorithmes de Fourier à des parties d'images expérimentales, en utilisant le niveau de bruit comme critère de résolution. Ces algorithmes permettent de déterminer automatiquement la résolution et sont simples à mettre en œuvre. Nous étudions la résolution locale dans une ligne de balayage et nous caractérisons le dispositif expérimental. Nous discutons la notion de résolution en champ proche.

\section{Introduction}

The Near Field Optical setups provide high resolved images. The lateral resolution is far beyond the Rayleigh criterion $(\lambda / 20$ is obtained [1-3]). The main point is the understanding of the image formation in the near field setups. Actually, the images depend strongly on many experimental parameters. We will discuss this fact in the first section. The resolution in near field optics is usually determined by using test samples (gratings, biological samples, steps...) [4-6]. The resolution is often determined by examining the intensity curve [5] or the image Fourier spectrum in the case of periodic samples $[7,8]$. On the opposite, theoretical studies describe the image formation with simulated images [9-14]. In that context, the resolution in near field optics was studied [15]. In this paper, we apply some methods that are well known in Scanning Electron Microscopy. Actually, the correlation functions and the phase spectrum are used for improving the signal-to-noise ratio in electron microscopy [16]. We use the Fourier spectrum of parts of the scanning lines that are delimited by a moving window 
to characterize locally and automatically the near field images and the setups. In this study, we choose the noise preponderance as resolution criterion. Effectively, the modulation transfer function (MTF) is very difficult to be defined in near field optics because the distance between the tip and the sample and the experimental conditions of illumination are critical parameters in the image formation $[1,17]$. We will underline the related artefacts in Scanning Near Field Microscopies in the first part.

A recent technique based on the detection of the noise in images, by spline fitting, enables to test the method [18]. That technique uses the shear-force and the optical image to calculate the resolution. In this study, we will investigate the same optical image in order to verify the current algorithm accuracy.

The images that we processed are recorded with a Reflection Scanning Near Field Microscope. In this configuration, the tip is used to light up the sample and simultaneously to detect the diffracted light in the reflection SNOM $[7,19]$. The images are series of such scanning lines. The scanned line is composed of light intensity values that are recorded at each tip position. The tip scans the sample at a constant distance from the sample by using shear-force feedback. In the next part, we will introduce the algorithm of the image processing and the artefacts related to near field microscopy.

\section{Optical Near Field Artefacts: the Problem of Resolution}

In the case of classical microscopy the MTF involves the knowledge of a few experimental parameters [20,21]. On the other hand, scanning near field microscopies could imply strong interaction between the tip and the sample. Moreover, images are strongly dependent on the scanned tip properties, since the small distance between tip and sample involves electromagnetic interactions. Indeed, some works related to near field microscopies intend to find a transfer function (MTF) or a point spread function (psf) assuming a linear information transfer from object to detector [1]. They take into account either the tip size [22] or theoretical aspect of imaging [11,14,17,23-27]. Some are developed around statistical processing of experimental data $[28,29]$, or the setup cut-off frequency determination [18]. These techniques and accurate models of the near field imagery can lead to solutions of the inverse scattering reconstruction problem $[17,26,30,31]$. In particular cases, the inverse scattering reconstruction problem was solved. Above all, varying the properties of the incoming light can change drastically images of the same sample. Moreover, the tip size, the tip collection angle, the tip manufacturing, the tip position can influence the images [13,32-37]. Contrast inversion in regards of the roughness may even be observed [10,12,38-40]. This effect can be due to the detection of index variations in the sample, to interferences effects in Near Field, or to the tip properties. To illustrate the problem of the image formation, we consider the near field above a dielectric rectangular grating [32]. The grating is illuminated by a plane wave. At first we suppose normal incidence and we look at the intensity at a few nanometers from the surface of the grating. That is a very crude model of a SNOM in transmission, but it takes the advantages of showing pure near-field effects. If the incident light is polarized and if the electric field is perpendicular to the grating grooves, enhancement of the edges of the grating grooves is observed (like "rabbit ears"). This effect could introduce artefacts by applying crudely the conventional algorithms to calculate the resolution. Then if the incident angle is over the critical angle like in the STOM (Scanning tunneling Optical Microscope) configuration, one can consider two polarizations. The first one is the s polarization (electric field parallel to the grooves) and the second one is the p polarization (electric field in the plane of incidence). If the polarization is $\mathrm{s}$, the near field image of the grating shows a smoothed profile whereas if polarization is $\mathrm{p}$ the edges of grating profile 
are better resolved and the image profile is more correlated to the sample profile. Thus, the resolution can strongly depend on the polarization. Moreover, contrast inversion can appears in s polarization, if the lateral size of the sample is over $\lambda$ over two. This effect is due to the properties of the transfer of the light in relation with the electromagnetic filtering induced by the polarization transfer matrices in a multilayered structure. The s polarization leads to a low pass filtering and the p polarization to a high pass filtering. Above all, the image formation involves low pass filtering related to the distance of detection. This exponentially decrease is demonstrated and is used in the last section, for the resolution calculation.

Thus, it seems to be difficult to introduce resolution considerations in regard of the topography on one hand and of the optical index profile, on the other hand. Pure topographical variations induce variations of the optical path like index inhomogeneities. On the other hand index inhomogeneities diffract like topographic variations. Thus, the resolution can only be determined in images, independently of the physical principles of image formation, except in special studies or in particular cases (study of the same sample with the same tip by varying the tip to sample distance for example). Consequently, we use the noise preponderance in the signal Fourier spectrum as resolution criterion. This takes the advantage of being applicable to each part of scanning line as well as the whole image, to characterize the setup. In this paper, we will investigate only one scanning line. In the next part we introduce the classical method of spectrogram to investigate the resolution.

\section{Image Processing: the Method}

SNOM images contain a lot of information on the sample. We know that the images are dependent on the topography, on the index profile of the sample, on the distance between the tip and the sample and on the illumination conditions. Thus resolution determination is a complex physical problem. The resolution study can be applied to a particular scanning line or can lead to a whole characterization of the setup [18]. We suppose that we can neglect the other filtering properties by assuming the exponential decrease to be stronger than the other filters. Thus by spline fitting, only exponential decay remains in the processed data. This assumption is related to the fact that the tip can be passive probe and acts like a dipolar center that detects the near-field. The transfer function can thus be defined as low pass exponential decay. This assumption is valid in the case of no-polarized incident light and of normal incidence of the light, like in the reflection SNOM configuration where the tip illuminates the sample and simultaneously detects the diffracted light.

The main point of the method is the study of the signal to noise ratio over the spatial frequencies. Thus, we compute the spectrograms along each scanning line. It consists of multiplying a window Win $(x)$ (rectangular or Gaussian) by the investigated scanning line $\mathrm{SL}(x)$ and of computing the Fast Fourier Transform (FFT) over the window. The process is iterated by sliding the window along the scanning line. This process gives rise to a $3 \mathrm{D}$ map of power spectra as a function of the window location. The abscissa are the harmonic order $p$ of the Fourier frequencies. If the window contains 51 pixels, then $-25<p<+25$. The corresponding spatial frequency is given by :

$$
f=\frac{p}{L[\operatorname{Win}(x)]}
$$

with $L[\mathrm{Win}(x)]$ being the size of the window in the direct space (in pixels for example). Usually, the generalized frequency is used. By this way, $0<p_{\mathrm{G}}<0.5$, it is related to $p$ by $p_{\mathrm{G}}=p / N$, where $N$ is the amount of pixels. 
Because Fast Fourier Transform (FFT) is used, results are more accurate if a preprocessing is applied:

- the line is joined with the reversed same line,

- the consequent junction is smoothed by spline (typically 11 pixels with spline coefficient $0.01)$.

This simple treatment enables to avoid the aliasing and the artefacts due to the junction. Doubling the size of the line implies doubling the number of Fourier harmonics, but the sampling step remains constant. In the following, the complete scanning line is 128 pixels and $7 \mu \mathrm{m}$ long.

The location of the spatial frequency where noise becomes preponderant is then easy to determine. The associated number of the Fourier frequency is $p_{\lim }$ and the resolution is given by:

$$
R=\frac{7 \mu \mathrm{m}}{128} \frac{L[\mathrm{Win}(x)]}{p_{\mathrm{lim}}}=\frac{7 \mu \mathrm{m}}{128 f_{\mathrm{lim}}}=\frac{7 \mu \mathrm{m}}{128} \frac{1}{p_{\mathrm{G}_{\mathrm{lim}}}}
$$

$p_{\mathrm{G}}$ is the generalized Fourier frequency. The location where noise becomes preponderant is calculated with the help of the spline fitting of the Fourier spectrum of $\mathrm{Win}(x) \cdot \mathrm{SL}(x)$. The local resolution can be studied by using small $L[\operatorname{Win}(x)]$. The process of the whole scanning line or of a collection of scanning lines can lead to an approach of apparatus characterization. To determine apparatus features, statistical samples should be used. In the present case, the sample and the apparatus are characterized. The near field image formation invokes a low-pass filtering of Fourier frequencies $[17,38]$. This filter is an exponential decrease and is related to the distance of detection. Thus we suppose that this filtering effect leads to a global exponential decrease of the amplitude of Fourier frequencies in the scanning line. The fitting of the Fourier spectrum can average the contribution of the sample features to the spectrum. Therefore, fitting helps us to study setup regardless of the sample.

In the case of periodical sample, the method must be carefully applied because of the periodical peaks that appear in the Fourier spectrum. The parameter of the spline fitting must be lower than in the case of the non periodical sample. In this study, the investigate scanning line corresponds to a non periodical sample. The spline fitting parameter is greater than 0 (mean square fitting) and lower than 1 (interpolation function).

The resolution criterion is deduced from above: In the case of noise preponderance, the fitted spectrum is no longer decreasing. The determination of the resolution is calculated by finding the point where the slope changes.

\section{Results}

Figure 2 shows the spectrogram associated to the scanning line in Figure 1, with $L[\operatorname{Win}(x)]=$ 100 pixels. Figure 3 shows the associated phase in scanning line. The three zones located by arrows contain strong variation of the phase in images. The size of these "dots" are 3 pixels to 4 pixels long. This figure can furnish to information on resolution: the size of the dots corresponds to the minimum information on the phase. Thus, resolution appears to reach at least $200 \mathrm{~nm}$.

Figure 1 shows the resolution we could determine by visual inspection. The zone 1 is the image of a cylindrical dot. The scanned line is $7 \mu \mathrm{m}$ long thus, resolution can be evaluated to $200 \mathrm{~nm}$. Figure 4 (resp. Fig. 5) is the multiple plot of the processed spectrum using $L[\mathrm{Win}(x)]=100$ pixels (resp. 50 pixels). In Figure 4, the spline parameter is 0.02 whereas in Figure 5, it is equal to 0.002 . In the left part of curves we observe a linear decrease. This fact 


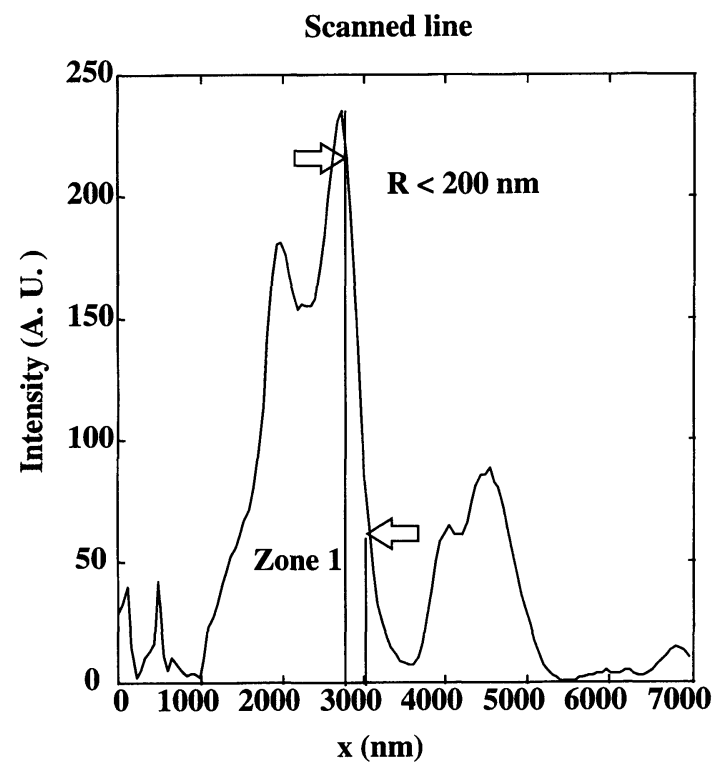

Fig. 1. - The scanning line we processed in this study (intensity). Zone 1 shows the image of a single cylindrical dot. The resolution can be evaluated by inspection of the shape of the profile: about $200 \mathrm{~nm}$. Abscissa are in $\mathrm{nm}$ and ordinate is in arbitrary units.

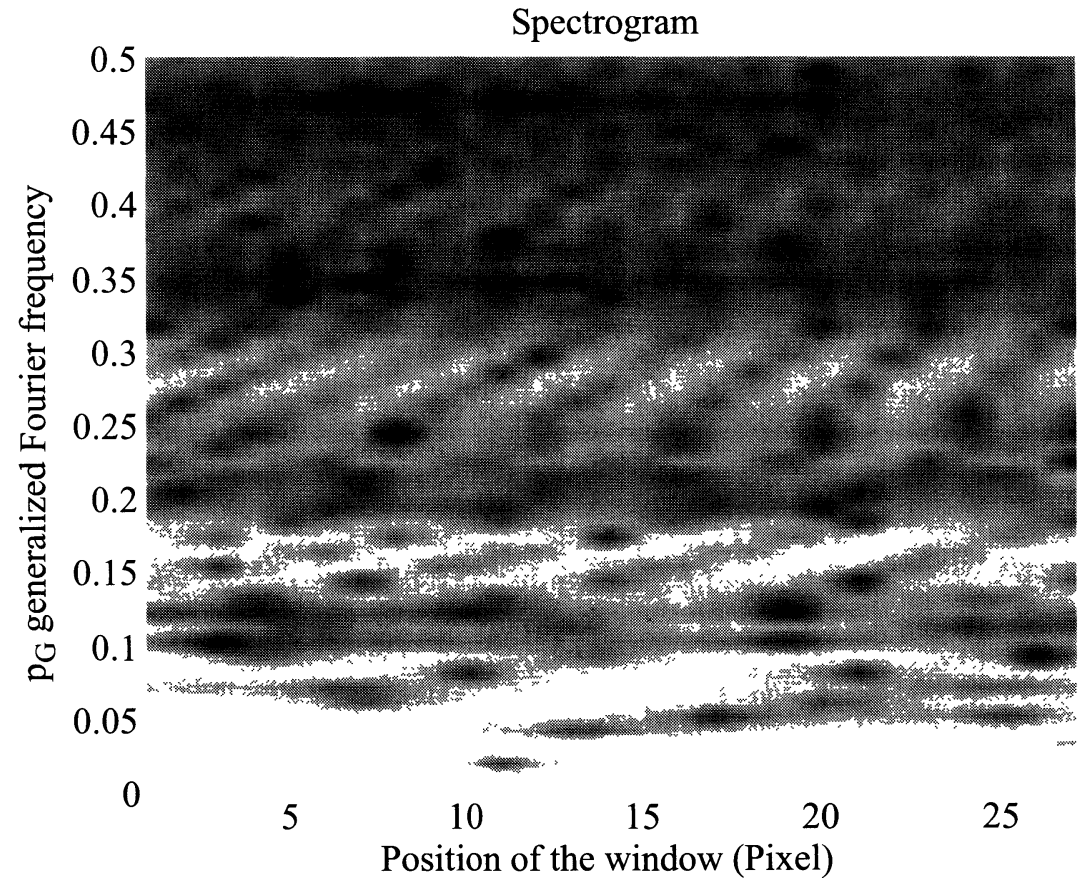

Fig. 2. - Logarithm of the Fourier spectrum (spectrogram). Abscissa is the position of the sliding window (pixels) with reference to the left of the scanned line plotted in Figure 1. The ordinate is the generalized frequency of the Fourier spectrum $\left(p_{\mathrm{G}}\right)$. 


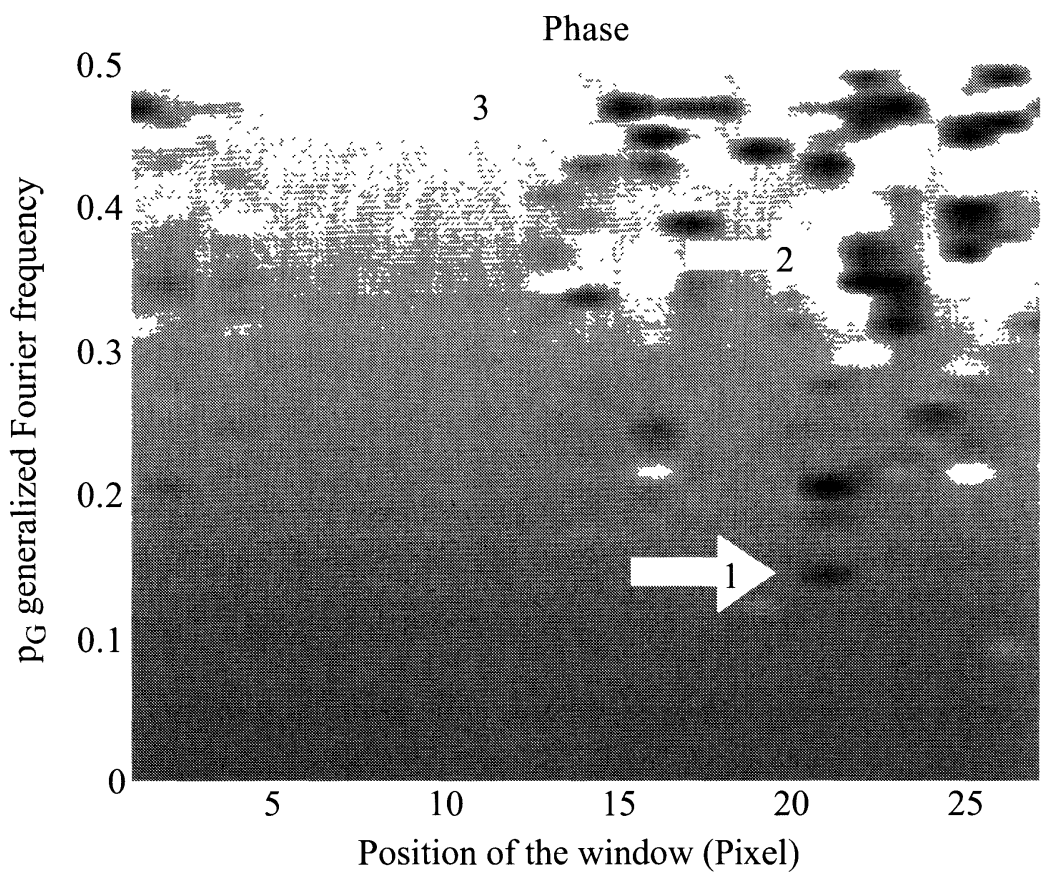

Fig. 3. - Phase deduced from the Fourier spectrum. The abscissa is the position of the sliding window (pixels) with reference to the left of the scanned line plotted in Figure 1. The ordinate is the generalized frequency of the Fourier spectrum $\left(p_{\mathrm{G}}\right)$. The zones 1,2 and 3 correspond to typical variations of the phase in the image. Their size ( $\sim 4$ pixels) is an evaluation of the resolution.

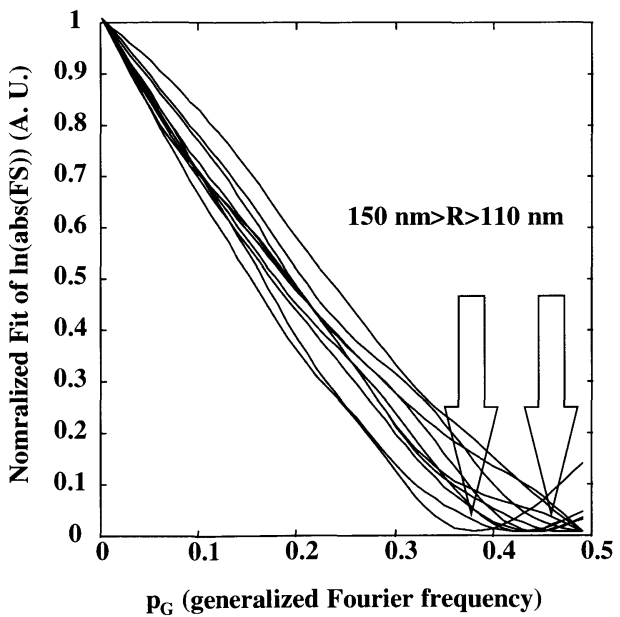

Fig. 4

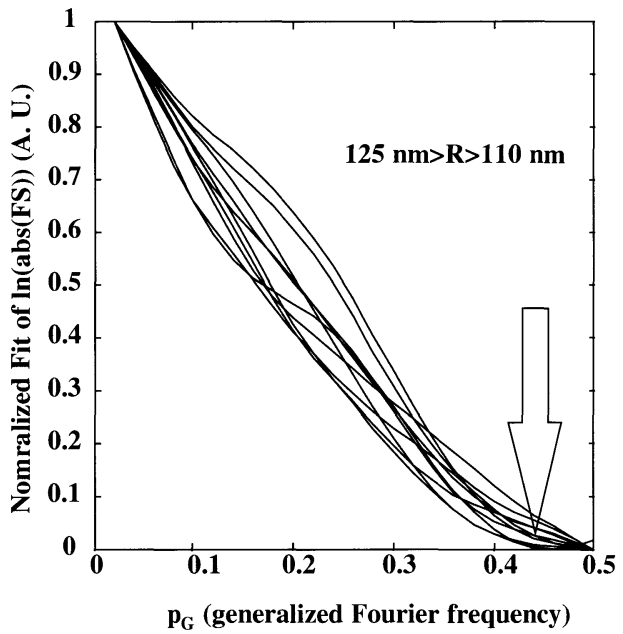

Fig. 5

Fig. 4. - Spline fitting of the logarithm of the Fourier spectrum. Abscissa are the generalized frequency in the Fourier spectrum $\left(p_{\mathrm{G}}\right)$. The ordinate is normalized. The spline fitting parameter is $0.02, L[\operatorname{Win}(x)]=100$ pixels. The resolution estimation is about $150 \mathrm{~nm}$.

Fig. 5. - Spline fitting of the logarithm of the Fourier spectrum. Abscissa are the generalized frequency in the Fourier spectrum $\left(p_{\mathrm{G}}\right)$. The ordinate is normalized. The spline fitting parameter is $0.002, L[\mathrm{Win}(x)]=50$ pixels. The resolution estimation is about $120 \mathrm{~nm}$. 
confirms the validity of spectrum exponential decrease described before. In the right part of the curves, we observe a change of the slope. The corresponding lateral resolutions is around $\lambda / 6$. If the window size is less, the resolution varies from one curve to the other. Thus, the local resolution is calculated. The local resolution is between $110 \mathrm{~nm}$ and $150 \mathrm{~nm}$. On the whole scanning line, we obtained $280 \mathrm{~nm}$ in reference [18]. The difference is due to the choice of the location of the sliding window. In this study, we retain only the 8 better resolved sliding windows. On the contrary, the global resolution given in reference [18] is calculated on the whole scanning line. Moreover, if the sliding window is smaller, the spline parameter must be smaller: compare Figures 4 and 5. In Figure 5, the fit shows the dependence of the spectrum on the scanning area specific properties. The low pass filtering is no more a pure exponential decay. The calculated resolution is better in this case, because the investigated area is smaller.

Above all, the choice of the spline parameter is not critical. As we shown in [18], there is a wide range of available spline parameter. A spline parameter belonging to [0.0001,0.05] is available. The same remark can be made for the smoothing of the junction (spline parameter: 0.01). The choice of the number of pixels that is used for this smoothing does not influence the results, if it is lower than the number of pixels of the whole line divided by 20 .

Finally, the method that we describe in this paper takes the advantage of being applicable to optical near field images, without needing corresponding Shear-Force image [18].

\section{Conclusion}

In this study, we use noise considerations to determine the resolution in Near Field Optics. When image contains only noise, the main physical filtering is drowned: the exponential decay vanishes. We applied Fourier algorithms, based on the spectrograms calculations. Then, we use spline fitting to calculate automatically the resolution in parts of scanning lines. The method takes advantage of being simple to be numerically implemented, and it furnishes objective results, without needing test sample or visual inspection. The method gives a global information on the scanning line window. The resolution obtained with the setup developed in Besançon is better than $\lambda / 6$. This result is bore out by using smaller scanning windows. Now, we are investigating the determination of unknown experimental parameter by using such methods. It should be interesting to apply this method to other near field microscopies where the images formation laws can be easily determined. Other methods, working in the direct space, could furnish more local information on the resolution, we will compare our algorithm to these methods. Another way consists in calculating the correlations between two series of data that are recorded in identical conditions [41]. That method could increase signal-to-noise ratio. We will use that method to enhance the resolution in SNOM images that are recorded with metal coated tip, where signal to noise ratio is very low.

\section{Acknowledgments}

This research has been supported by the "Institut des Microtechniques de Franche-Comté" of Besançon. I thank Noël Bonnet (Université de Champagne-Ardennes) for suggestions of improvement of the method. I thank Christian Pieralli (Université de Franche-Comté) for fruitful contribution to the problem of resolution and I am grateful to Olivier Bergossi (Université Technologique de Troyes) and Michel Spajer (Université de Franche-Comté) for experimental images providing and for fruitful discussions on the method. 


\section{References}

[1] Courjon D., Near-field imaging: some attempts to define an apparatus function, J. Microscopy 177 (1995) 180-185.

[2] Lacoste Th., Huser Th., Heinzelmann H. and Güntherrodt H.-J., Scanning near-field optical microscopes for high resolution imaging, in "Photons and Local Probes", Othmar Marti and Rolf Möller, Eds. (Kluwer Academic Publishers, Dordrecht, 1995) vol. 300 of NATO ASI Series E: Appl. Sci., pp. 123-132.

[3] Krenn J.R., Somitsch D., Gotschy W., Leitner A. and Aussenegg F.R., Near-field optical investigations on nanometric silver particles, in "Photons and Local Probes", Othmar Marti and Rolf Möller, Eds. (Kluwer Academic Publishers, Dordrecht, 1995) vol. 300 of NATO ASI Series E: Appl. Sci., pp. 181-187.

[4] Van Hulst N.F., Segerink F.B. and Bölger B., High resolution imaging of dielectric surfaces with an evanescent field optical microscope, Opt. Commun. 87 (1992) 212-218.

[5] Masheri R.U., Tatsumi H.Y., Katayama and Ohtsu M., Observation of subcellular nanostructure of single neurons with an illumination mode photon scanning tunneling microscope, Optics Commun. 120 (1995) 325-334.

[6] de Fornel F., Salomon L., Adam P., Bourillot E. and Goudonnet J.P., Resolution of the photon scanning tunneling microscope: influence of physical parameters, Ultramicroscopy 42-44 (1992) 422-429.

[7] Bergossi O., Spajer M. and Schiavone P., Local index measurements by reflection near field optical microscopy, in "Near Field Optics - 3" (Kluwer Academic Publishers, Brno, Czech Republic, 1995) pp. 179-180.

[8] Maheswari R.U., Kadono H. and Ohtsu M., Power spectral analysis for evaluating optical near-field images of $20 \mathrm{~nm}$ gold particles, Optics Commun. 131 (1996) 133-142.

[9] Girard Ch. and Spajer M., Model for reflection near-field optical microscopy, Appl. Opt. 29 (1990) 3726-3733.

[10] Girard Ch. and Bouju X., Self-consistent study of dynamical and polarization effects in near field optical microscopy, J. Opt. Soc. Am. B 9 (1992) 298-305.

[11] Vigoureux J.M., Depasse F. and Girard Ch., Superresolution of near-field optical microscopy defined from properties of confined electromagnetic waves, Appl. Opt. 31 (1992) 3036-3045.

[12] Van Labeke D. and Barchiesi D., Probes for scanning tunneling optical microscopy: A theoretical comparison, J. Opt. Soc. Am. A 10 (1993) 2193-2201.

[13] Barchiesi D. and Van Labeke D., Une modélisation de l'influence de la métallisation des objets et de la forme des sondes en SNOM, Microsc. Microanal. Microstruct. 5 (1994) $1-10$.

[14] Cho K., Ohfuti Y. and Arima K., Study of scanning near-field optical microscopy (SNOM) by nonlocal response theory, Jpn J. Appl. Phys. 34 (1994) 267-270.

[15] Garcia N. and Nieto-Vesperinas M., Theory for the apertureless near-field optical microscope: Image resolution, Appl. Phys. Lett. 66 (1995) 3399-3400.

[16] Bonnet N., On the use of correlation functions for improving the image signal-to-noise ratio, Optik 80 (1988) 103-106.

[17] Carminati R. and Greffet J.-J., Two-dimensional numerical simulation of the photon scanning tunneling microscope. Concept of transfer function, Optics Commun. 116 (1995) 316-321; erratum in Optics Commun. 120 (1995) 371. 
[18] Barchiesi D., Bergossi O., Spajer M. and Pieralli C., Image resolution in reflection scanning near-field optical microscopy (R-SNOM) using shear-force (ShF) feedback: Characterization using spline and fourier spectrum, Appl. Opt. 36 (1997) 2171-2177.

[19] Spajer M., Courjon D., Sarayedine K., Jalocha A. and Vigoureux J.M., Microscopie en champ proche par réflexion, Revue Phys. Appl. 26 (1989) 111-112.

[20] Goodman J.W., Introduction to Fourier optics (Mc Graw-Hill, New-York, 1968).

[21] Sheppard C.J.R., Gu M. and Mao X.Q., Three-dimensional coherent transfer function in a reflection-mode confocal scanning microscope, Optics Commun. 81 (1991) 281-284.

[22] Reiss G., Schneider F., Vancea J. and Hoffman H., Scanning tunneling microscopy on rough surfaces: Deconvolution of constant current images, Appl. Phys. Lett. 57 (1990) 867-869.

[23] Vigoureux J.M. and Courjon D., Detection of nonradiative fields in light of the Heisenberg uncertainty principle and the Rayleigh criterion, Appl. Opt. 31 (1992) 3170-3177.

[24] Kann J.L., Milster T.D., Froehlich F.F., Ziolkowski R.W. and Judkins J.B., Linear behavior of near-field optical system, J. Opt. Soc. Am. A 12 (1995) 1677-1682.

[25] Nakano T. and Kawata S., Numerical analysis of the near-field diffraction pattern of a small aperture, J. Mod. Opt. 39 (1992) 645-661.

[26] Garcia N. and Nieto-Vesperinas M., A direct solution to the inverse scattering problem in near-field optical microscopy: Object structure reconstruction, in "Photons and Local Probes", Othmar Marti and Rolf Möller, Eds. (Kluwer Academic Publishers, Dordrecht, 1995) vol. 300 of NATO ASI Series E: Appl. Sci., pp. 47-57.

[27] Madrazo A. and Nieto-Vesperinas M., Surface structure and polariton interactions in the scattering of electromagnetic waves from a cylinder in front of conducting grating: theory for the reflection photon scanning tunneling microscope, J. Opt. Soc. Am. A 13 (1996) 785-795.

[28] Pieralli C., Statistical estimation of point spread function applied to scanning near-field optical microscopy, Optics Commun. 108 (1994) 203-208.

[29] Pieralli C., Estimation of point-spread functions and modulation-transfer functions of optical devices by statistical properties of randomly distributed surfaces, Appl. Opt. 33 (1994) 8186-8193.

[30] Greffet J.-J., Santenac A. and Carminati R., Surface profile reconstruction using near-field data, Optics Commun. 116 (1995) 20-24.

[31] Nieto-Vesperinas M. and Madrazo A., A theoretical study of near-field interactions with local probes, in "Photons and Local Probes", Othmar Marti and Rolf Möller, Eds. (Kluwer Academic Publishers, Dordrecht, 1995) vol. 300 of NATO ASI Series E: Appl. Sci., pp. 35-46.

[32] Barchiesi D., Modélisation Des Microscopes Optiques En Champ Proche : STOM et SNOM. Optimisation et Spectroscopie., Ph.D. thesis, Université de Franche-Comté (Feb. 23 1993).

[33] Barchiesi D. and Van Labeke D., Application of mie scattering of evanescent waves to scanning optical tunneling microscopy theory, J. Mod. Opt. 40 (1993) 1239-1254.

[34] Barchiesi D. and Van Labeke D., Scanning tunneling optical microscopy (STOM): Theoretical study of polarization effects with two models of tip, in "Near Field Optics", D. W. Pohl and D. Courjon, Eds. (Kluwer Academic Publishers, Dordrecht, 1993) vol. 242 of NATO ASI Series E: Appl. Sci., pp. 179-188.

[35] Bozhevolnyi S., Keller O. and Xiao M., On the resolution limit in near-field optical microscopy, in "Near Field Optics", D. W. Pohl and D. Courjon, Eds. (Kluwer Academic Publishers, Dordrecht, 1993) vol. 242 of NATO ASI Series E: Appl. Sci., pp. 229-238. 
[36] Novotny L., Pohl D.W. and Regli P., Near-field, far-field and imaging properties of the 2D aperture snom, Ultramicroscopy 57 (1995) 180-188.

[37] Barchiesi D., A 3-d method for calculating near-field diffracted by nano-structures: Application to thin-coated nano-sources, Optics Commun. 126 (1996) 7-13.

[38] Van Labeke D. and Barchiesi D., Scanning-tunneling optical microscopy: a theoretical macroscopic approach, J. Opt. Soc. Am. A 9 (1992) 732-739.

[39] Baida F., Mered C. and Courjon D., Microscope à double détection: STOM-AFM, principe et résultats, Microsc. Microanal. Microstruct. (1994) 19-29.

[40] Barchiesi D. and Van Labeke D., PSTM: An alternative to measure local variation of optical index, Microsc. Microanal. Microstruct. 5 (1994) 435-446.

[41] Bonnet N., Lebonvallet S., El Hila H., Colliot G. and Beorchia A., Quantitative evaluation of the signal to noise ratio and of its improvement (or degradation) by digital filters, $J$. Phys. III France 1 (1991) 1349-1358. 